\title{
Sub-Arcsecond near-IR Imaging and Long-Slit Spectroscopy of NGC 7027
}

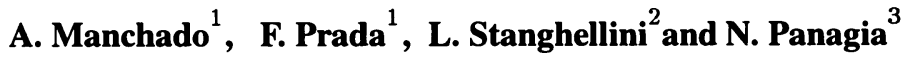 \\ Instituto de Astrofísica de Canarias Osservatorio Astronomico di Bologna 1; Space Telescope \\ Science Institute $2 ; 3$
}

We report sub-arcsecond narrow band imaging in the lines of $\mathrm{H}_{2} 2.1218 \mu \mathrm{m}, \mathrm{Br} \gamma 2.16$ $\mu \mathrm{m},[\mathrm{Fe} \mathrm{II}] 1.664 \mu \mathrm{m}$ of the Planetary Nebulae NGC 7027. Data were obtained at the $3.5 \mathrm{~m}$ telescope at Calar Alto Observatory (Spain), using MAGIC. A false color image was produced by combining these three narrow band images. Thus, and due to extraordinary seeing (0".6 arcsecond) conditions, allow us to reveal the morphology of the excited molecular and ionized gas. The $\mathrm{Br} \gamma$ and the [Fe II] images allowed us to study the structure of the ionized gas at slightly different excitation levels, according to the ionization potentials of the ions involved in the corresponding transitions $(13.6 \mathrm{eV}$ to ionize hydrogen, versus $17.4 \mathrm{eV}$ for iron ionizaton). Since NGC 7027 is a high excitation nebula, the iron is readily double-ionized in the hotter regions, thus what we really see in the [Fe II] image is the low-intermediate excitation zones. By studying the [Fe II] and $\mathrm{Br} \gamma$ images together we infer that: the (non-reddened) low-ionization gas morphology consists of a regular, smooth ring reminder of a projected cylindrical structure. In addition to a brightness level that is at least thirty times the brightness of the maximum [Fe II] emission, the ionized hydrogen image exhibit a strong brightness gradient between the mentioned ring and the outer regions, and the emission is comparatively negligible outside the ring-like feature of about $12 \times 10$ arcsec (the measures refer to the major and minor axes of the ellipse-like shape). The [Fe II] morphology is less so ring-like and regular, and it seems to indicate smaller scale structure, such as clumps and filaments, at the resolution scale-level. The dishomogeneity in the [Fe II] emission could be ascribed to gradients in the elemental distribution, as well (and more probably so) as to the plasma temperature and density inhomogeneities. The $\mathrm{H} 2$ molecular image completely embeds the ionized gas zones, up to a size of about $16 \times 11$ arcsec. The smaller size refers here to the waist of the markedly bipolar shape, similar to a projected peanut; the larger size is the dimension of the two main lobes. The highest brightness through the molecular filters corresponds to the inner ring, slightly less extended than the ionized one, and to an outer envelope that is not seen in the ionized gas, and has a maximum diagonal extension of about 24 arcsec. Spatially resolved low resolution spectra ( $\mathrm{R} 200$ ) were obtained in the $\mathrm{H}$ and $\mathrm{K}$ band. 